\title{
Atividade anti-helmíntica de Spigelia anthelmia L. no controle de parasitos gastrintestinais de Gallus gallus
}

Anthelmintic activity of Spigelia anthelmia L. in the control of gastrointestinal parasites of Gallus gallus

\author{
G. F. Vita ${ }^{1 *}$; I. Ferreira ${ }^{1}$; M.A.V. Da Costa Pereira ${ }^{2}$; A. Sanavria ${ }^{1}$; R. De C.M. \\ Aurnheimer ${ }^{3}$ \\ ${ }^{1}$ Universidade Federal Rural do Rio de Janeiro (UFRRJ), 23890-000, Rio de Janeiro - RJ, Brasil \\ ${ }^{2}$ Universidade Estadual do Norte Fluminense Darcy Ribeiro (UENF), 28013-602, Campos dos Goytacazes - RJ, Brasil \\ ${ }^{3}$ Médica Veterinária, Centro Universitário Anhaguera de Niterói (UNIAN), Niterói-RJ, Brasil \\ *gilmarferreiravita@yahoo.com.br
}

(Recebido em 20 de dezembro de 2018; aceito em 24 de março de 2019)

\begin{abstract}
A pesquisa foi desenvolvida no Departamento de Epidemiologia e Saúde Pública da Universidade Federal Rural do Rio de Janeiro (UFRRJ) e Setor de Parasitologia Animal da Universidade Estadual do Norte Fluminense Darcy Ribeiro (UENF), no período de 2015 a 2018. O objetivo foi testar in vitro e in vivo a eficácia da planta medicinal Spigelia anthelmia Linnaeus, nas formas fitoterápica e homeopática, como meios alternativos para o controle de endoparasitos de Gallus gallus Linnaeus (1758), um sério problema que afeta a criação e desempenho de aves domésticas, ocasionando morte quando muito intenso, retardo de crescimento, redução de índice de conversão alimentar e aumento na suscetibilidade às doenças infecciosas. Para o experimento in vitro, foi realizado teste de inibição de eclosão de ovos e teste de inibição do desenvolvimento larvar; e, para o experimento in vivo, foi realizado teste de redução da contagem de ovos nas fezes. As metodologias utilizadas para execução dos testes foram creditadas pela World Association for the Advancement of Veterinary Parasitology (WAAVP), organização referência para testes anti-helmínticos. S. anthelmia demonstrou eficácia nos experimentos in vitro e in vivo, com valores máximos de 55,00\% (homeopatia) e 67,00\% (fitoterapia), no teste de inibição de eclosão de ovos, 82,00\% (homeopatia) e 75,00\% (fitoterapia), no teste de inibição do desenvolvimento larvar, e 81,82\% (homeopatia) e 75,00\% (fitoterapia), no teste de redução da contagem de ovos nas fezes. A planta apresentou valor superior ao produto tradicional utilizado (Febendazol) no experimento in vitro, ficando dentro dos índices propostos pela WAAVP, Ministério da Agricultura do Brasil e Organização Mundial da Saúde, como indicativos de eficácia.

Palavras-chave: planta medicinal, endoparasitoses, aves domésticas.
\end{abstract}

The research was developed at the Departamento de Epidemiologia e Saúde Pública of the Universidade Federal Rural do Rio de Janeiro (UFRRJ) and Setor de Parasitologia Animal of the Universidade Estadual do Norte Fluminense Darcy Ribeiro (UENF), from 2015 to 2018. The objective was to test in vitro and in vivo the efficiency of the medicinal plant Spigelia anthelmia Linnaeus, in phytotherapeutic and homeopathic forms, as an alternative way to control endoparasites of the Gallus gallus Linnaeus (1758), a serious problem that affects the breeding and performance of domestic bird, causing death when very intense, growth retardation, reduction of feed conversion rate and increase in susceptibility to infectious diseases. For the in vitro experiment, egg hatching inhibition test and larval development inhibition test were performed; and, for the in vivo experiment, fecal egg count reduction test was performed. The methodologies used were supported by the World Association for the Advancement of Veterinary Parasitology (WAAVP), reference to anthelmintic tests. S. anthelmia demonstrated efficacy in vitro and in vivo experiments, with maximum values of $55.00 \%$ (homeopathy) and $67.00 \%$ (phytotherapy), in the egg hatching inhibition test, $82.00 \%$ (homeopathy) and $75.00 \%$ (phytotherapy), in the larval development inhibition test, and $81.82 \%$ (homeopathy) and $75.00 \%$ (phytotherapy), in the fecal egg count reduction test. The plant presented higher value than the traditional product used (Febendazole) in the in vitro experiment, being within the indices proposed by WAAVP, Ministry of Agriculture of Brazil and World Health Organization, as indicative of efficacy.

Keywords: medicinal plant, endoparasites, domestic birds. 


\section{INTRODUÇÃO}

A infecção por nematoides gastrintestinais é considerada um sério problema sanitário na criação de aves domésticas no Brasil. Em muitos casos a avicultura alternativa de frangos e galinhas caipiras é um fator de subsistência para agricultores, que retiram daí sua principal fonte de renda. No entanto, as endoparasitoses limitam a produção de seus plantéis, ocasionando prejuízos econômicos e consequentemente de qualidade de vida. O país, considerado o terceiro maior produtor mundial de galináceos, abrange cerca de 1.4 bilhões de exemplares, e dentro deste cenário, a criação de aves caipiras apresenta uma forte contribuição, estando presente em torno de $80,00 \%$ das propriedades rurais [1].

Dentre os nematoides gastrintestinais desencadeadores de infecções que acometem as aves domésticas, destacam-se as espécies Ascaridia galli, Heterakis gallinarum e Capillaria, mais frequentemente encontradas em inquéritos. Elas são responsáveis por inúmeras patologias, entre elas, hemorragias, enterites, obstrução intestinal, perfuração da mucosa, necrose e morte quando muito intensas $[2,3]$.

Contrapondo os meios tradicionais, principalmente à administração de bases químicas, que contribui para o aparecimento de resíduos nos produtos animais, novas alternativas tem surgido para o controle dessas parasitoses, com destaque para a utilização de plantas da "medicina popular". A fitoterapia e a homeopatia surgem para promover esse controle, ofertando aos criadores uma metodologia com proposta de eliminar os resíduos e diminuir os malefícios ocasionados às aves [4, 5].

A fitoterapia e a homeopatia, como medicamentos com finalidade profilática, curativa, paliativa e fins de diagnóstico, tiveram sua recomendação pela Organização Mundial da Saúde, a partir de 1978 e 2002, respectivamente. Ambas as terapias são pouco implantadas no mercado veterinário brasileiro, onde apenas $1,00 \%$ do valor movimentado pelo segmento fitoterápico é voltado para esse setor. Com relação ao segmento homeopático, esta pesquisa é uma das pioneiras, principalmente em relação às endoparasitoses de aves domésticas $[6,7,8]$.

No Brasil, diversas plantas são utilizadas no controle de verminoses através de conhecimento empírico. Nesta pesquisa procurou-se investigar cientificamente a planta medicinal Spigelia anthelmia Linnaeus (Erva Lombrigueira), cuja atividade anti-helmíntica remonta à medicina indígena, que usava as raízes e folhas da planta como vermífugo e inseticida, e atualmente, tem sido estudada como inibidora de infecções por nematoides gastrintestinais em diferentes espécies animais $[9,10]$.

Sendo assim, o objetivo desta pesquisa foi testar in vitro e in vivo a eficácia da planta medicinal S. anthelmia, nas formas fitoterápica e homeopática, como meios alternativos para o controle de endoparasitos de Gallus gallus Linnaeus, 1758 (Galinha Caipira). Justifica-se a pesquisa pelo fato de existir um elevado índice de resíduos químicos nos produtos originários desta espécie, pela resistência aos fármacos usados de forma indiscriminada e pelas grandes perdas dos rebanhos ocasionadas por doenças parasitárias.

\section{MATERIAL E MÉTODOS}

A pesquisa foi conduzida no Departamento de Epidemiologia e Saúde Pública, da Universidade Federal Rural do Rio de Janeiro (UFRRJ), localizada no município de Seropédica, estado do Rio de Janeiro, e Setor de Parasitologia Animal, Laboratório de Sanidade Animal, Hospital Veterinário, Centro de Ciências e Tecnologias Agropecuárias, da Universidade Estadual do Norte Fluminense Darcy Ribeiro (UENF), localizada no município de Campos dos Goytacazes, estado do Rio de Janeiro, no período de 2015 a 2018.

Constou de dois experimentos, um in vitro, teste de inibição de eclosão de ovos e teste de inibição do desenvolvimento larvar, e outro in vivo, teste de redução da contagem de ovos nas fezes, onde se avaliou a eficácia da planta $S$. anthelmia, nas formas homeopática e fitoterápica.

Os extratos da planta foram obtidos comercialmente no Laboratório Dr. Faria E.M. Mello Ltda, sendo na forma fitoterápica como tintura mãe e na forma homeopática em baixa dinamização hahnemanniana (CH6), ambas diluídas em solução alcoólica a 70,00\%. 
Para a pesquisa foram empregadas galinhas caipiras com aproximadamente 24 semanas de vida e peso vivo médio de $2 \mathrm{~kg}$, criadas em sistema extensivo, com infecção parasitária natural, sem administração anterior de anti-helmínticos em período de três meses [11].

As galinhas foram provenientes de criações particulares do município de Seropédica, estado do Rio de Janeiro. Foram acondicionadas em 12 boxes padronizados (3,00 x 2,00 m), com 10 animais cada, cobertos com telha e área de exposição à luz natural, temperatura ambiente, separados por arame galvanizado, com poleiros adequados sem arestas cortantes e piso coberto com maravalha. A alimentação das aves constou de milho, ração e verduras, por três vezes ao dia, num total médio de aproximadamente $120 \mathrm{~g} / \mathrm{dia}$. As aves receberam água ad libitum $[12,13]$.

Nos dias da realização dos testes, seja para o experimento in vitro ou in vivo, o piso sob a área dos boxes foi forrado com lona plástica às 05:00 horas da manhã, com recolhimento do material fecal às 06:00 horas. Este foi acondicionado em potes plásticos, devidamente identificados, mantidos sob refrigeração $\left(2\right.$ a $\left.8^{\circ} \mathrm{C}\right)$ e encaminhados ao laboratório para realização das análises em no máximo três horas, a contar do momento em que se fez a forragem $[11,14]$.

As metodologias utilizadas nos dois experimentos, in vitro e in vivo, foram baseadas em Coles et al. (1992) [11] e Hubert \& Kerbouef (1992) [15], e foram assim realizadas:

\section{Experimento in vitro}

\section{Técnica de suspensão de ovos de nematoides}

Para realização do experimento in vitro (teste de inibição de eclosão de ovos e teste de inibição do desenvolvimento larvar), foi realizada uma suspensão de ovos com utilização de 40 galinhas dispostas em quatro boxes, com 10 animais cada. Um montante de $100 \mathrm{~g}$ de fezes frescas, divididas em $25 \mathrm{~g}$ por boxe, foram coletadas e enviadas ao laboratório, onde foram suspensas em água e limpas de restos orgânicos por filtração através de tamises de 140, 70 e $30 \mu \mathrm{m}$, sendo os ovos recolhidos num último tamise de $16 \mu \mathrm{m}$. Os ovos foram ainda mais limpos de restos orgânicos por centrifugação em sulfato de magnésio (densidade 1:10) durante cinco minutos a $1000 \mathrm{rpm}$. $\mathrm{O}$ sobrenadante foi filtrado através de tamises de $70 \mu \mathrm{m}$ e $30 \mu \mathrm{m}$ e os ovos foram lavados com água e recolhidos em um tamise de $16 \mu \mathrm{m}$ [15]. A suspensão elaborada constou ao final de aproximadamente $75 \mathrm{ml}$.

\section{Teste de inibição de eclosão de ovos}

Para este teste foram utilizados 12 tratamentos: cinco na forma fitoterápica, com volumes de $0,250 \mathrm{ml}, 0,200 \mathrm{ml}, 0,150 \mathrm{ml}, 0,100 \mathrm{ml}$ e $0,050 \mathrm{ml}$ de tintura mãe; cinco na forma homeopática com volumes de $0,250 \mathrm{ml}, 0,200 \mathrm{ml}, 0,150 \mathrm{ml}, 0,100 \mathrm{ml}$ e 0,050 $\mathrm{ml}$ de CH6; um controle negativo, com volume de $0,250 \mathrm{ml}$ de água; e, um controle positivo, com volume de $0,010 \mathrm{ml}$ de solução de Febendazol (Provermin ${ }^{\circledR}$ - Indubras, Brasil), conforme estabelecido pelo laboratório. Na realização do teste, $2 \mathrm{ml}$ da suspensão de ovos foram disponibilizados para cada tratamento, sendo colocados em poços de placas de cultivo (cada placa: 12 poços x $6 \mathrm{ml}$ ). Acrescentou-se os extratos das plantas, homeopático e fitoterápico, o controle negativo (água) e controle positivo (Febendazol), nos volumes estipulados. Incubou-se a $27^{\circ} \mathrm{C}$ por 48 horas e após esse período duas gotas de solução Lugol's iodine foram adicionadas paralisando a incubação dos ovos. Todas as larvas de segundo estádio recém-eclodidas em cada poço foram contadas. O teste foi realizado em triplicata [11].

\section{Teste de inibição do desenvolvimento larvar}

Neste teste, 12 tratamentos foram utilizados: cinco na forma fitoterápica, com volumes de 0,250 $\mathrm{ml}, 0,200 \mathrm{ml}, 0,150 \mathrm{ml}, 0,100 \mathrm{ml}$ e $0,050 \mathrm{ml}$ de tintura mãe; cinco na forma homeopática com volumes de $0,250 \mathrm{ml}, 0,200 \mathrm{ml}, 0,150 \mathrm{ml}, 0,100 \mathrm{ml} \mathrm{e} 0,050 \mathrm{ml}$ de CH6; um controle negativo, com volume de $0,250 \mathrm{ml}$ de água; e, um controle positivo, com volume de $0,010 \mathrm{ml}$ de solução de Febendazol, conforme estabelecido pelo laboratório. Para o procedimento do teste, $2 \mathrm{ml}$ da suspensão de ovos, juntamente com $2 \mathrm{~g}$ de fezes provenientes de galinhas livres de nematoides gastrintestinais, foram disponibilizados para cada tratamento, sendo colocados em tubos de $15 \mathrm{ml}$ 
de Clayton Lane. As fezes foram utilizadas como meio nutritivo. Os tubos foram fechados e colocados em uma incubadora à temperatura de $23^{\circ} \mathrm{C}$ por 48 horas, período necessário para a eclosão dos ovos e nascimento das larvas de segundo estádio. Neste momento adicionou-se os extratos homeopático e fitoterápico da planta, o controle negativo (água) e o controle positivo (Febendazol), nos volumes mencionados. Novamente os tubos foram levados à incubadora na temperatura de $23^{\circ} \mathrm{C}$, esperando por cinco dias. Após esse período, foi acrescentado três gotas de solução de Lugol's iodine e realizada a contagem de larvas de terceiro estádio. Para evitar a proliferação de fungos, $10 \mathrm{mg}$ de Amphotericine B (Fungizone ND; Squibb) foram adicionadas aos tubos no início do experimento. O teste foi realizado em triplicata [15].

\section{Experimento in vivo}

\section{Teste de redução da contagem de ovos nas fezes}

A metodologia utilizada para este teste seguiu Coles et al. (1992) [11] e foi assim definida: os animais foram distribuídos aleatoriamente em grupos controles negativo (água) e positivo (Febendazol) e tratados (fitoterápico e homeopático), em quatro boxes, com 10 animais cada, conforme recomendação do Ministério da Agricultura, Pecuária e Abastecimento (1997) [16]. Um mínimo de 30 gramas de fezes foi coletado de cada boxe. As amostras foram conduzidas ao laboratório para a contagem dos ovos (dia 1), que foi realizada em triplicata. Este teste foi repetido por três vezes, com a utilização de 40 galinhas em cada, totalizando 120 aves.

No procedimento tratamento: em cada teste, os animais foram tratados por três dias alternados. Quando do controle positivo (Febendazol), por deposição na ração, no volume de 1,2 g para cada galinha, conforme estabelecido pelo fabricante, e quando dos tratamentos homeopático e fitoterápico, por deposição em água, no volume de $15 \mathrm{ml}$ para cada galinha, adequação proveniente do volume que apresentou maior eficácia nos testes in vitro de inibição de eclosão de ovos e de inibição do desenvolvimento larvar $(0,250 \mathrm{ml}$ do produto), e calculada com base no peso diário de alimento fornecido ao animal e consequentemente em trânsito gastrintestinal, assim sendo:

\section{VCin vivo $=\frac{\text { Volume mais eficaz nos testes in vitro } \mathrm{x} \text { alimento diário ingerido }}{2^{*}}$}

* $2 \mathrm{ml}$ (ou g) da suspensão de ovos controlada pelo volume mais eficaz nos testes in vitro.

Doze dias após o tratamento, as amostras fecais foram coletadas e o número de ovos novamente contados, em triplicata. No procedimento da contagem de ovos nas fezes, foi utilizada a técnica de McMaster modificada [17].

Os ovos e larvas dos endoparasitos encontrados foram identificados segundo chaves de identificação e características morfológicas estabelecidas por Vicente et al. (1995) [18] e McDougald (1997) [19], e observados à luz da microscopia óptica, com aumento de 10x, 40x e 100x.

A análise estatística dos dados obtidos das contagens parasitológicas foi realizada através da análise de variância (ANOVA) e complementada pelo teste de Tukey $(\mathrm{p}<0,05)$ [20]. A eficácia dos testes in vitro, inibição de eclosão de ovos e inibição do desenvolvimento larvar, foi determinada segundo Bizimenyera et al. (2006) [21], de acordo com a seguinte equação:

Onde,

$$
\% \mathrm{E}=100(1-\text { Pteste/Pcontrole })
$$

Pteste $=$ número de larvas de segundo estádio (L2) observadas (no caso do teste de inibição de eclosão de ovos nos grupos tratados), ou número de larvas que desenvolveram para larvas de terceiro estádio (L3) observadas (no caso do teste de inibição do desenvolvimento larvar nos grupos tratados) e,

Pcontrole $=$ respectivo número no controle negativo (água).

Esta pesquisa foi submetida à Comissão de Ética na Pesquisa da Universidade Federal Rural do Rio de Janeiro, sob o número de processo 000.909.2016, ficando estabelecido que a mesma 
encontra-se de acordo com os preceitos da Lei $\mathrm{n}^{\mathrm{o}} 11794$, de 08 de outubro de 2008 , do Decreto $\mathrm{n}^{\mathrm{o}}$ 6.899, de 15 de julho de 2009, e com as normas editadas pelo Conselho Nacional de Experimentação Animal (CONCEA), no âmbito dos princípios éticos e do bem estar animal.

\section{RESULTADOS}

Foram observados endoparasitos pertencentes aos gêneros Ascaridia (14,00\%), Capillaria $(60,00 \%)$ e Heterakis $(26,00 \%)$.

Após a realização do teste de inibição de eclosão de ovos, foi observado um maior percentual de inibição no volume fitoterápico e homeopático de $0,250 \mathrm{ml}, 67,00 \%$ e $55,00 \%$, respectivamente, acima do valor observado no controle positivo $(52,00 \%)$. Na análise estatística verificou-se que as médias do tratamento homeopático e fitoterápico no volume de $0,250 \mathrm{ml}$ do extrato da planta, e do controle positivo, diferiram significativamente da média do controle negativo, o que demonstra uma eficácia desses produtos/volumes frente à inibição de eclosão de ovos (p < 0,05; GL = 11-24; $\mathrm{F}=4,1346)($ Tabela 1).

Tabela 1: Número médio de larvas de segundo estádio (L2) de endoparasitos de Gallus gallus, encontrados após a aplicação dos tratamentos fitoterápico e homeopático da planta medicinal Spigelia anthelmia, controle positivo (Febendazol) e controle negativo (água), com seus devidos volumes, e percentual de inibição de eclosão de ovos.

\begin{tabular}{|c|c|c|c|c|c|}
\hline \multirow{2}{*}{ Tratamentos/Volumes } & \multicolumn{3}{|c|}{$\begin{array}{l}\text { Larvas (L2) } \\
\text { (repetiçõos) }\end{array}$} & \multirow{2}{*}{$\begin{array}{l}\text { Larvas (L2) } \\
\text { (média geral) }\end{array}$} & \multirow{2}{*}{$\begin{array}{l}\text { Inibição de eclosão } \\
\text { de ovos (\%) }\end{array}$} \\
\hline & $1^{\underline{a}}$ & $2^{\mathrm{a}}$ & $3^{\mathrm{a}}$ & & \\
\hline \multicolumn{6}{|l|}{ Homeopatia } \\
\hline $0,250 \mathrm{ml}$ & 24 & 26 & 25 & $25 \mathrm{a}^{*}$ & 55,00 \\
\hline $0,200 \mathrm{ml}$ & 46 & 42 & 26 & $38 \mathrm{~b}$ & 32,00 \\
\hline $0,150 \mathrm{ml}$ & 44 & 37 & 36 & $39 \mathrm{c}$ & 30,00 \\
\hline $0,100 \mathrm{ml}$ & 61 & 15 & 33 & $36,33 \mathrm{~d}$ & 34,00 \\
\hline $0,050 \mathrm{ml}$ & 41 & 33 & 43 & $39 \mathrm{e}$ & 30,00 \\
\hline \multicolumn{6}{|l|}{ Fitoterapia } \\
\hline $0,250 \mathrm{ml}$ & 16 & 17 & 22 & $18,33 \mathrm{f}$ & 67,00 \\
\hline $0,200 \mathrm{ml}$ & 21 & 22 & 18 & $20,33 \mathrm{~g}$ & 64,00 \\
\hline $0,150 \mathrm{ml}$ & 34 & 30 & 27 & $30,33 \mathrm{~h}$ & 46,00 \\
\hline $0,100 \mathrm{ml}$ & 33 & 43 & 23 & $33 \mathrm{i}$ & 41,00 \\
\hline $0,050 \mathrm{ml}$ & 43 & 31 & 41 & $38,33 \mathrm{j}$ & 31,00 \\
\hline \multicolumn{6}{|c|}{ Controle positivo (Febendazol) } \\
\hline $0,010 \mathrm{ml}$ & 24 & 29 & 27 & $26,66 \mathrm{k}$ & 52,00 \\
\hline \multicolumn{6}{|l|}{ Controle negativo (água) } \\
\hline $0,250 \mathrm{ml}$ & 49 & 56 & 61 & 55,33 afgk & - \\
\hline
\end{tabular}

* Médias de tratamentos seguidas por letras iguais diferem significativamente entre si pelo teste de Tukey (p $<0,05)$.

Para o teste de inibição do desenvolvimento larvar, os resultados demonstraram uma maior eficácia do tratamento homeopático nos volumes de $0,250 \mathrm{ml}(82,00 \%)$ e $0,150 \mathrm{ml}(85,00 \%)$, ambos superiores ao melhor resultado do tratamento fitoterápico no volume de $0,250 \mathrm{ml}(75,00 \%)$ e do controle positivo $(65,00 \%)$. Na análise estatística diversos foram os tratamentos/volumes que evidenciaram eficácia do extrato da planta na inibição do desenvolvimento larvar, frente ao controle negativo, menciona-se aqui os volumes de 150 e $250 \mathrm{ml}$ do tratamento homeopático, $250 \mathrm{ml}$ do tratamento fitoterápico, e o controle positivo $(\mathrm{p}<0,05 ; 11-24 ; \mathrm{F}=6,6337)$ (Tabela 2).

Tabela 2: Número médio de larvas de terceiro estádio (L3) de endoparasitos de Gallus gallus, encontrados após a aplicação dos tratamentos fitoterápico e homeopático da planta medicinal Spigelia anthelmia, 
controle positivo (Febendazol) e controle negativo (água), com seus devidos volumes, e percentual de inibição do desenvolvimento larvar.

\begin{tabular}{|c|c|c|c|c|c|}
\hline \multirow{2}{*}{ Tratamentos/Volumes } & \multicolumn{3}{|c|}{$\begin{array}{l}\text { Larvas (L3) } \\
\text { (repetições) }\end{array}$} & \multirow{2}{*}{$\begin{array}{l}\text { Larvas (L3) } \\
\text { (média geral) }\end{array}$} & \multirow{2}{*}{$\begin{array}{c}\text { Inibição do } \\
\text { desenvolvimento } \\
\text { larvar }(\%)\end{array}$} \\
\hline & $1^{\mathrm{a}}$ & $2^{\mathrm{a}}$ & $3^{\mathrm{a}}$ & & \\
\hline \multicolumn{6}{|l|}{ Homeopatia } \\
\hline $0,250 \mathrm{ml}$ & 11 & 8 & 4 & $7,66 \mathrm{a}^{*}$ & 82,00 \\
\hline $0,200 \mathrm{ml}$ & 16 & 10 & 11 & $12,33 \mathrm{~b}$ & 71,00 \\
\hline $0,150 \mathrm{ml}$ & 4 & 9 & 7 & $6,66 \mathrm{c}$ & 85,00 \\
\hline $0,100 \mathrm{ml}$ & 21 & 16 & 28 & $21,66 \mathrm{~d}$ & 49,00 \\
\hline $0,050 \mathrm{ml}$ & 6 & 13 & 22 & $13,66 \mathrm{e}$ & 68,00 \\
\hline \multicolumn{6}{|l|}{ Fitoterapia } \\
\hline $0,250 \mathrm{ml}$ & 11 & 16 & 6 & $11 \mathrm{f}$ & 75,00 \\
\hline $0,200 \mathrm{ml}$ & 17 & 19 & 28 & $21,33 \mathrm{~g}$ & 50,00 \\
\hline $0,150 \mathrm{ml}$ & 30 & 20 & 26 & 25,33 ach & 41,00 \\
\hline $0,100 \mathrm{ml}$ & 14 & 31 & 17 & $20,66 \mathrm{i}$ & 52,00 \\
\hline $0,050 \mathrm{ml}$ & 20 & 44 & 23 & 29 acj & 32,00 \\
\hline \multicolumn{6}{|c|}{ Controle positivo (Febendazol) } \\
\hline $0,010 \mathrm{ml}$ & 11 & 20 & 14 & $15 \mathrm{k}$ & 65,00 \\
\hline \multicolumn{6}{|l|}{ Controle negativo (água) } \\
\hline $0,250 \mathrm{ml}$ & 53 & 35 & 39 & 42,33 abcefk & - \\
\hline
\end{tabular}

* Médias de tratamentos seguidas por letras iguais diferem significativamente entre si pelo teste de Tukey (p $<0,05)$.

Na Tabela 3 pode-se observar o número médio de ovos por grama de fezes de endoparasitos de G. gallus, constatado durante as três realizações do experimento in vivo, para o dia 1 , inicial à aplicação, e para o dia 12, ao término da aplicação, e o número médio total das três realizações. Contabilizou-se ao final de todas as realizações, uma queda no número médio total de ovos de 183,33 para 33,33 (= 150) no tratamento homeopático, 200 para $50(=150)$ no tratamento fitoterápico, e 150 para $16,66(=133,34)$ no tratamento controle positivo.

Tabela 3: Número médio de ovos de endoparasitos de Gallus gallus, por grama de fezes, no dia 1 e 12, observado após três repetições, e após três realizações do teste de redução da contagem de ovos nas fezes, referente aos tratamentos fitoterápico e homeopático da planta medicinal Spigelia anthelmia, controle positivo (Febendazol) e controle negativo (água).

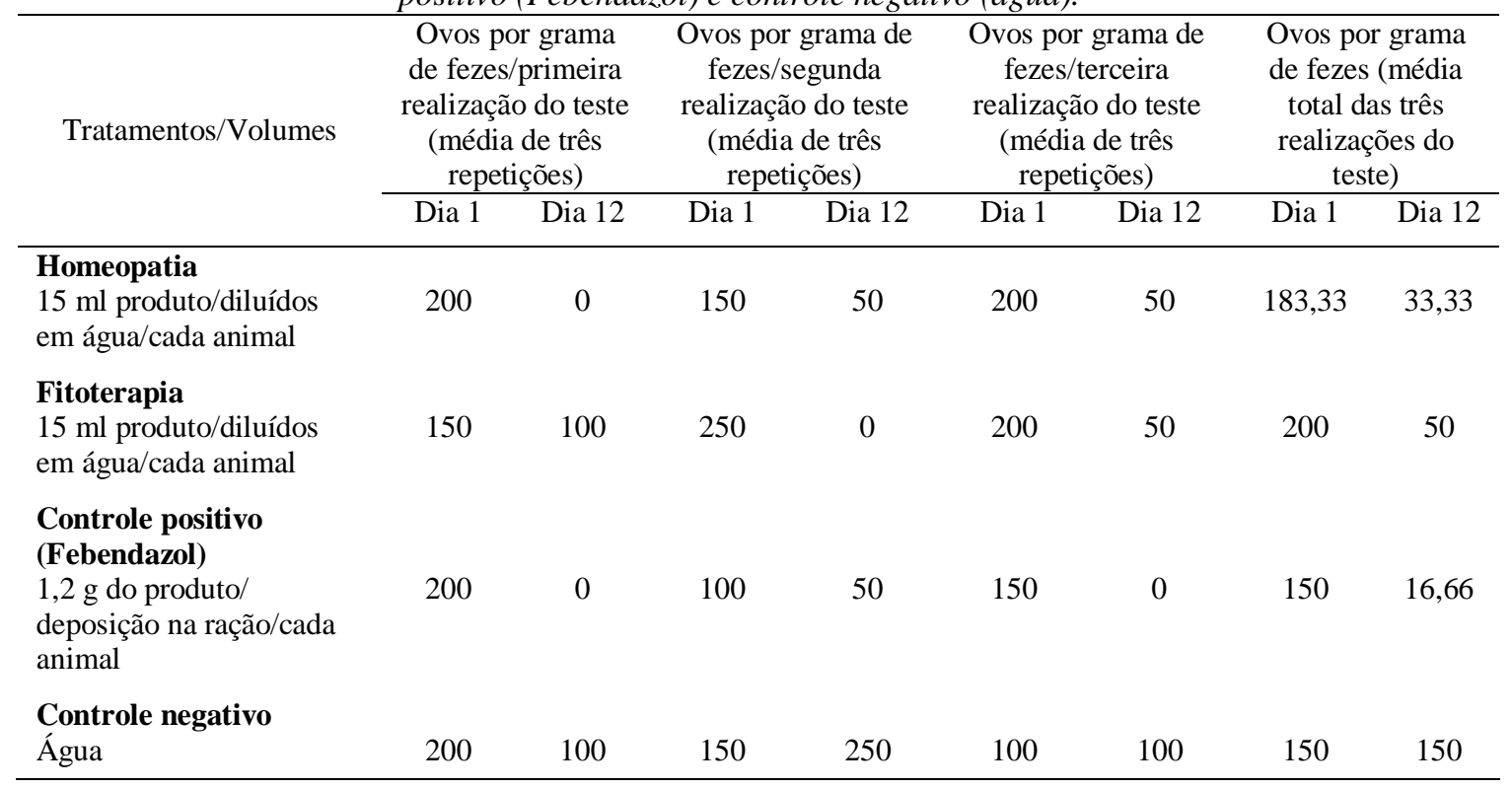


Os resultados observados no experimento in vivo confirmaram um maior percentual de redução da contagem de ovos nas fezes no controle positivo $(88,90 \%)$, ficando os produtos homeopático $(81,82 \%)$ e fitoterápico $(75,00 \%)$ com percentuais bem próximos. A análise estatística comprovou que não houve uma redução significativa entre as médias da contagem de ovos por grama de fezes antes e após a administração dos tratamentos ( $\mathrm{p}<0,05 ; \mathrm{GL}=1-3 ; \mathrm{F}=0,7879)$, e que também não existiu uma diferença significativa entre as médias intertratamentos $(\mathrm{p}<0,05 ; \mathrm{GL}=3-3 ; \mathrm{F}=$ 8,8730 ), inclusive com o controle negativo (Tabela 4).

Tabela 4: Número médio total de ovos de endoparasitos de Gallus gallus, por grama de fezes, no dia 1 e

12, observado depois das três realizações do teste de redução da contagem de ovos nas fezes, referente aos tratamentos fitoterápico e homeopático da planta medicinal Spigelia anthelmia, controle positivo (Febendazol) e controle negativo (água), e percentual de redução da contagem de ovos nas fezes.

\begin{tabular}{|c|c|c|c|c|}
\hline \multirow[t]{2}{*}{ Tratamentos/Volumes } & \multicolumn{2}{|c|}{$\begin{array}{l}\text { Ovos por grama de fezes } \\
\text { (média total das três } \\
\text { realizações do teste) }\end{array}$} & \multirow[t]{2}{*}{$\begin{array}{c}\text { Média } \\
\text { Intertratamentos }\end{array}$} & \multirow{2}{*}{$\begin{array}{c}\text { Redução da } \\
\text { contagem de } \\
\text { ovos nas fezes } \\
(\%)\end{array}$} \\
\hline & Dia 1 & Dia 12 & & \\
\hline $\begin{array}{l}\text { Homeopatia } \\
15 \mathrm{ml} \text { produto/diluídos em água/cada } \\
\text { animal }\end{array}$ & 183,33 & 33,33 & $108,33 a^{*}$ & 81,82 \\
\hline $\begin{array}{l}\text { Fitoterapia } \\
15 \mathrm{ml} \text { produto/diluídos em água/cada } \\
\text { animal }\end{array}$ & 200 & 50 & $125 \mathrm{~b}$ & 75,00 \\
\hline $\begin{array}{l}\text { Controle positivo (Febendazol) } \\
1,2 \mathrm{~g} \text { do produto/deposição na } \\
\text { ração/cada animal }\end{array}$ & 150 & 16,66 & $83,33 \mathrm{c}$ & 88,90 \\
\hline $\begin{array}{l}\text { Controle negativo } \\
\text { Âgua }\end{array}$ & 150 & 150 & $133,33 \mathrm{~d}$ & 0,00 \\
\hline Média antes e após tratamentos & $162,49 \mathrm{a}$ & $62,49 \mathrm{~b}$ & & \\
\hline
\end{tabular}

\section{DISCUSSÃO}

Os gêneros observados nesta pesquisa, Ascaridia, Capillaria e Heterakis, são também relatados na maioria dos estudos que versam sobre parasitismo gastrintestinal de aves domésticas [2, 22, 23, $24,25,26]$, podendo ser considerados como os de maior ocorrência em infecções parasitárias dessas espécies.

Vita et al. (2014) [25] mencionou que a ocorrência de endoparasitos em G. gallus de regimes extensivo ou semi-extensivo, provavelmente está relacionada ao contato direto com o solo, utilização de fontes hídricas não tratáveis, e sobrevivência prolongada de ovos desses parasitos no meio ambiente [27, 28, 29]. Nesta pesquisa constatou-se que onde as aves habitavam circulavam outras espécies animais (silvestres ou domésticas), e que a falta de higiene, chão enlameado, contato com as fezes, água não tratada, foram fatores que favoreceram a proliferação das doenças parasitárias.

De acordo com a classificação do índice de eficácia proposto pela World Association for the Advancement of Veterinary Parasitology (WAAVP), organização de onde provieram os ensaios in vitro de inibição de eclosão de ovos e in vivo de redução da contagem de ovos nas fezes, um produto seria efetivo se apresentasse ação igual ou acima de 60,00\% [30]. Já quanto aos critérios da Organização Mundial da Saúde e do Ministério da Agricultura do Brasil, um produto seria considerado como eficiente, se obtivesse patamares acima de $80,00 \%$ e $75,00 \%$, respectivamente $[31,32]$.

Seguindo os critérios de tais organizações, a planta S. anthelmia demonstrou no experimento in vitro, índices máximos não efetivo (forma homeopática - 55,00\%) e efetivo (forma fitoterápica $67,00 \%$ ), no teste de inibição de eclosão de ovos; e, índices máximos efetivos nas formas homeopática $(85,00 \%)$ e fitoterápica $(75,00 \%)$, no teste de inibição do desenvolvimento larvar. Já quanto ao ensaio in vivo, evidenciou índices efetivos em ambas as formas, homeopática $(81,82 \%)$ 
e fitoterápica $(75,00 \%)$, no teste de redução da contagem de ovos nas fezes. O grupo controle positivo atingiu índices efetivos nos testes do ensaio in vitro, com valor máximo de $65,00 \%$, e nos testes do ensaio in vivo $(88,90 \%)$.

De acordo com a classificação do índice de eficácia proposto pelas mesmas organizações, podese afirmar que a planta $S$. anthelmia atingiu índices efetivos em ambos os ensaios (in vitro e in vivo). O produto natural nos segmentos in vitro teve valores maiores que o produto tradicional (Febendazol), e próximo aos valores do mesmo no segmento in vivo.

O Febendazol é um anti-helmíntico de amplo espectro, comumente usado na medicina veterinária. Tem uma eficácia superior a 90,00\% contra nematoides gastrintestinais de aves, o que foi demonstrado em trabalhos realizados por diversos autores [33, 34, 35]. Assim, constatar que a planta utilizada nesta pesquisa tem valores próximos ou superiores ao deste produto conceituado, é certamente confirmar seu poder para utilização no tratamento das endoparasitoses aviárias.

Raros são os trabalhos que utilizam $S$. anthelmia no controle de endoparasitos de aves, sejam domésticas ou silvestres, como fitoterapia ou homeopatia, mas existem outros que confirmam sua ação anti-helmíntica. Ademola et al. (2007) [36] estudando o efeito in vivo da planta sobre nematoides gastrintestinais de ovinos, verificaram uma redução percentual na contagem de ovos por grama de fezes após 12 dias de tratamento, num patamar de 76,00\% para Haemonchus sp., 79,80\% para Oesophagostomum sp., 76,00\% para Strongyloides sp. e 48,00\% para Trichuris sp. Assis et al. (2003) [37] em sua pesquisa in vitro confirmaram a efetividade no controle de nematoides de ovinos e caprinos, com inibição de $100,00 \%$ da incubação dos ovos e 81,20\% do desenvolvimento larvar.

A pesquisa recomenda o estudo das substâncias presentes nos extratos de cada planta, pois é possível suspeitar que algumas isoladamente sejam as responsáveis pelo efeito anti-helmíntico. Ntalli et al. (2010) [38] estudando o efeito isolado de terpenos, dentre eles, o geraniol e o timol, contra o nematoide Meloidogyme incognita, concluíram que esses compostos foram mais ativos individualmente do que misturados. Portanto, testes com o alcaloide rianodina, presente em maior quantidade na planta desta pesquisa, além das outras substâncias, podem oferecer resultados mais confiáveis, quando ajustados aos testes realizados. Os resultados podem ser mais promissores se as substâncias forem testadas isoladamente [39].

Nesta pesquisa não foi realizada uma avaliação de toxicidade da planta, por entender que a mesma quando ministrada em baixas doses e períodos curtos é desprovida de toxicidade significativa [40]. Mesmo assim, uma validação toxicológica necessitará ser certificada para que a planta possa ser inserida como metodologia de controle de nematoides gastrintestinais de galinhas caipiras por criadores em suas propriedades. Dessa forma, sugere-se estudos futuros que embasem tal afirmativa.

A análise estatística realizada na pesquisa não demonstrou diferenças significativas entre as médias do experimento in vivo, diferentemente das altas significâncias observadas no experimento in vitro nos testes de inibição de eclosão de ovos e desenvolvimento larvar, o que não inviabiliza a ação da planta $S$. anthelmia, uma vez que a análise levou em consideração as médias entre os tratamentos e não os dados dos percentuais de redução, que atingiram valores acima de $80,00 \%$ de eficácia.

A transposição do volume de $0,250 \mathrm{ml}$ dos produtos fitoterápico e homeopático, considerado o de maior eficácia no controle dos endoparasitos no âmbito geral, para o ensaio in vivo da redução de contagem de ovos nas fezes, teve seu cálculo realizado encima do peso do alimento fornecido ao animal diariamente, isto é, quantidade do bolo fecal diário circulante. Vita et al. $(2014,2015)$ $[5,25]$ trabalhando com a planta medicinal Chenopodium ambrosioides, utilizou mesma metodologia para transposição do volume laboratorial para aplicação in vivo, e obteve resultados de 100,00\% de eficácia do produto natural, sobre os endoparasitos. Também foi utilizada a forma de administração sugerida pelo fabricante da base tradicional, que era oferecer o produto durante três dias alternados. A transposição foi eficaz, pois demonstrou índices de eficácia da planta na redução de contagem de ovos nas fezes, nos valores de 70,00\% na forma homeopática e $81,00 \%$ na forma fitoterápica. Acredita-se que volumes mais elevados que $0,250 \mathrm{ml}$, ajustados para o ensaio in vivo, irão revelar maiores eficácias.

Ressalta-se que as aves tratadas com a planta medicinal $S$. anthelmia, seja em qualquer forma utilizada, apresentaram maior ganho de peso, melhor postura e aparência. Culminando em cerca de 
$20,00 \%$ a mais na produção de ovos, frente àquelas que fizeram uso do produto controle positivo e negativo; tornando-se assim, uma eficiente alternativa para profissionais e criadores que buscam uma melhor qualidade de vida para seus animais, produtos sem resíduos químicos, ambiente mais limpo e maiores ganhos financeiros [5, 25].

Trabalhos que versam sobre controle de nematoides e tratamentos com anti-helmínticos naturais em aves são muito escassos; no Brasil pouco se dá importância a esse tipo de assunto, provavelmente pela vida curta que frangos de cortes possuem, passando pela fase de engorda de pintinho a adulto, num ciclo de aproximadamente 47 dias [25]. Mas há de considerar, que uma boa vermifugação desse plantel poderia trazer ainda mais ganhos econômicos ao produtor, diminuindo o índice de mortalidade e a ocorrência de doenças. Além disso, deve-se pensar naquele produtor rural, que faz da venda de ovos seu sustento, e que mantém seu animal durante anos; sem esquecer do criador de aves ornamentais e outros que domesticam suas aves. Parasitoses internas podem causar problemas respiratórios, perda de peso, retardo no desenvolvimento, podendo levar as aves à morte.

\section{CONCLUSÃO}

Os gêneros de endoparasitos identificados nesta pesquisa foram Ascaridia $(14,00 \%)$, Heterakis $(26,00 \%)$ e Capillaria $(60,00 \%)$.

No experimento in vitro, $S$. anthelmia apresentou eficácia sobre os endoparasitos encontrados na forma fitoterápica $(67,00 \%)$, do teste de inibição de eclosão de ovos, e nas formas homeopática $(85,00 \%)$ e fitoterápica $(75,00 \%)$, do teste de inibição do desenvolvimento larvar.

No experimento in vivo, $S$ anthelmia demonstrou eficácia em ambas as formas, homeopática $(81,82 \%)$ e fitoterápica $(75,00 \%)$, do teste de redução da contagem de ovos.

\section{AGRADECIMENTOS}

À Coordenação de Aperfeiçoamento de Pessoal de Nível Superior (CAPES), pelo financiamento da pesquisa.

\section{REFERÊNCIAS BIBLIOGRÁFICAS}

1. Ibge. Produção da pecuária municipal 2017. Rio de Janeiro: Instituto Brasileiro de Geografia e Estatística; 2017. $8 \mathrm{p}$.

2. Ogbaje CI, Agbo EO, Ajanusi OJ. Prevalence of Ascaridia galli, Heterakis gallinarum and tapeworm infections in birds slaughtered in Makurdi Township. International Journal of Poult Science. 2012 Fev;11(2):103-7.

3. Taylor MA, Coop RL, Wall RL. Veterinary Parasitology. 4rd ed. New Jersey: Wiley-Blackwell; 2015. $1032 \mathrm{p}$.

4. Sobral FES, Brandão PA, Athayde ACR. Utilização de fitoterápicos no tratamento de parasitoses em galinhas caipira criadas em sistema semi-extensivo. Agropecuária Cientifica no Semiárido. 2010 Jan/Mar;6(1):1-6. Doi.org/10.30969/acsa.v6il.64.

5. Vita GF, Ferreira I, Da Costa Pereira MAV, Sanavria A, Aurnheimer RCM, Barbosa CG, Gallo SSM, Vasconcellos HVG. Eficácia de Chenopodium ambrosioides (Erva-de-santa-maria) no controle de endoparasitos de Coturnix japonica (Codorna japonesa). Pesquisa Veterinária Brasileira. 2015 Mai;35(5):424-30,doi:10.1590/S0100-736X2015000500006

6. Brasil. Práticas integrativas e complementares: plantas medicinais e fitoterapia na Atenção Básica, Brasília: Ministério da Saúde; 2012. 156 p.

7. Ozaki AT, Duarte PC. Fitoterápicos utilizados na medicina veterinária, em cães e gatos. Revista Pharmacia Brasileira. Infarma. 2006 Jun;18(11-12):17-25.

8. Ibiapina $\mathrm{WV}$, Leitão BP, Batista MM, Pinto DS. Inserção da fitoterapia na atenção primária aos usuários do SUS. Revista Ciência Saúde Nova Esperança. 2014 Jun;12(1):58-68.

9. Nery PS, Duarte ER, Martins ER. Eficácia de plantas para o controle de nematóides gastrintestinais de pequenos ruminantes: revisão de estudos publicados. Revista Brasileiras Plantas Medicinais. 2009 Jul;11(3):330-8,doi:10.1590/S1516-05722009000300016 
10. Ribeiro WLC, Andre WPP, Cavalcante GS, Araújo-Filho JV, Santos JML, Macedo ITF, Melo JV, Morais SM, Bevilaqua CML. Effects of Spigelia anthelmia decoction on sheep gastrointestinal nematodes. Small Rumin Research. 2017 Aug;153:146-52,doi:10.1016/j.smallrumres.2017.06.001

11. Coles GC, Bauer C, Borgsteede FHM, Geerts S, Klei TR, Taylor MA, Waller PJ. World association for the Advancement of Veterinary Parasitology (WAAVP) methods for the detection of anthelmintic resistance in nematodes of veterinary importance. Veterinary Parasitology. 1992 Sep;44(1-2):3544,doi:10.1016/0304-4017(92)90141-U

12. Brasil. Decreto-Lei n. ${ }^{0} 72-\mathrm{F}$, de 14 de abril de 2003. Estabelece as normas mínimas de proteção das galinhas poedeiras, bem como as normas relativas ao registro de estabelecimentos de criação daquela espécie. Diário da República, Brasília, v.2452, n.88, p.97-102, 2003.

13. Santos MW, Ribeiro AGP, Carvalho LS. Criação de galinha caipira. Para produção de ovos em regime semi-intensivo. Niterói: Programa Rio Rural, Secretaria de Estado de Agricultura, Pecuária, Pesca e Abastecimento, Superintendência de Desenvolvimento Sustentável; 2009. 30 p.

14. Opas. Manual veterinário de colheita e envio de amostras. Rio de Janeiro: PANAFTOSA/OPAS/OMS; 2010. 218 p.

15. Hubert J, Kerboeuf D. A microlarval development assay for the detection of anthelmintic resistance in sheep nematodes. Vet Rec. 1992 May;130(20):442-6,doi:10.1136/vr.130.20.442

16. Ministério da Agricultura, Pecuária e Abastecimento. Portaria n. ${ }^{\circ} 48$, de 12 de maio de 1997. Aprova o Regulamento Técnico para Licenciamento e/ou Renovação de Licença de Produtos Antiparasitários de Uso Veterinário. Diário Oficial [da] República Federativa do Brasil, Poder Executivo, Brasília, DF, 16 mai. 1997. Seção 1. p. 10165.

17. Hoffmann RP. Diagnóstico de parasitismo veterinário. Porto Alegre: Sulina; 1987. 156 p.

18. Vicente JJ, Rodrigues HO, Gomes DC, Pinto RM. Nematóides do Brasil. Parte IV: Nematóides de Aves. Revista Brasileira de Zoologia. 1995;12(1):1-273,doi:10.1590/S0101-81751995000500001

19. McDougald LR. Protozoa. In: Calnek BW, editor. Diseases of poultry. 20 ed. Iowa: Iowa State University Press; 1997. p. 865-83.

20. Vieira S. Introdução à bioestatística. 5. ed. Rio de Janeiro: Elsevier, 2016. 242 p.

21. Bizimenyera ES, Githiori JB, Eloff JN, Swan GE. In vitro activity of Peltophorum africanum Sond. (Fabaceae) extracts on the egg hatching and larval development of the parasitic nematode Trichostrongylus colubriformis. Vet Parasitol. 2006 Dec;142(3-4):33643,doi:10.1016/j.vetpar.2006.06.013

22. Gomes FF, Machado HHS, Lemos LS, Almeida LG, Daher RF. Principais parasitos intestinais diagnosticados em galinhas domésticas criadas em regime extensivo na municipalidade de Campos dos Goytacazes, RJ. Ciênc Anim Bras. 2009 Sep;10(3):818-22,doi:10.5216/cab.v10i3.1095

23. Marin-Gómez SY, Benavides-Mantaño JA. Parásitos en aves domésticas (Gallus domesticus) en el Noroccidente de Colombia. Vet. Zootec. 2007 Jan;1(2):43-51.

24. Squires S, Fisher M, Gladstone O, Rogerson S, Martin P, Martin S, Lester H, Sygall R, Underwood N. Comparative efficacy of flubendazole and a commercially available herbal wormer against natural infections of Ascaridia galli, Heterakis gallinarum and intestinal Capillaria spp. in chickens. Vet Parasitol. 2012 Apr;185(2-4):352-54,doi:10.1016/j.vetpar.2011.09.034

25. Vita GF, Ferreira I, Da Costa Pereira MAV, Azevedo JR, Sanavria A, Barbosa CG, Gallo SSM, Vasconcellos HVG. Eficácia de Chenopodium ambrosioides (Erva-de-santa-maria) no controle de endoparasitos de Gallus gallus (Galinha caipira). Pesq Vet Bras. 2014 Jan;34(1)3945,doi:10.1590/S0100-736X2014000100007

26. Siqueira GB, Marques SMT. Parasitos intestinais em galinhas caipiras da região metropolitana de Porto Alegre, RS. Pubvet. 2016 Set;10(9):690-95.

27. Cardozo SP, Yamamura MH. Parasitas em produção de frangos no sistema de criação tipo colonial/caipira no Brasil. Semina: Ciênc. Agrár. 2004 Jan/Mar;25(1):63-74,doi:10.5433/1679-0359.2004v25n1p63

28. Quadros RM, Wiggers SB, Paes MPV, Marques SMT. Prevalência de endo e ectoparasitos de galinhas caipiras em pequenas propriedades da região serrana de Santa Catarina. Pubvet. 2014 Dez;8(24):1-5.

29. Siqueira GB, Marques SMT. Parasitos intestinais em galinhas caipiras da região metropolitana de Porto Alegre, RS. Pubvet. 2016 Set;10(9):690-95.

30. Powers KG, Wood IB, Eckert J, Gibson T, Smitc HJ. World Association for the Advancement of Veterinary Parasitology (W.A.A.V.P.) - Guidelines for evaluating the efficacy of anthelmintics in ruminants (bovine and ovine). Vet Parasitol. 1982 Jul;10(4):265-84,doi:10.1016/0304-4017(82)90078-4

31. Brasil. Portaria n. ${ }^{\circ} 90$, de 4 de dezembro de 1989. Normas para produção, controle e utilização de produtos antiparasitários. Diário da República, Brasília, seção 1, col. 2, 1990.

32. Who. Atividade carrapaticida. Resistência e susceptibilidade a drogas. Disponível em: <http://www.who.com>. Acesso em: 10 jan. 2007. 
33. Montalvo-Aguilar X, López Arellano ME, Vázquez-Prats V, Liébano Hernández E, Mendonza de Gives P. Resistencia antihelmíntica de nematodos gastroentéricos en ovinos a febendazol e ivermectina en la región noroeste del estado de Tlaxcala. Téc Pecu Méx. 2006 Jan;44(1):81-90.

34. Carlos CZ, Nunes PF, Silva T, Silva ARC. Mentha crispa e fembendazol no tratamento de parasitoses: um estudo clínico. Pubvet. 2011 Out;5(39):1-18.

35. Ssenyonga GSZ. Efficacy of fenbendazole against helminth parasites of poultry in Uganda. Trop Anim Health Pro. 1982 Sep;14(3):163-6,doi:10.1007/BF02242148.

36. Ademola IO, Fagbemi BO, Idowu SO. Anthelmintic activity of Spigelia anthelmia extract against gastrointestinal nematodes of sheep. Parasitol Res. 2007 Jun; 101(1):63-9, doi:10.1007/s00436-006-04440

37. Assis LM, Bevilaqua CML, Morais SM, Vieira LS, Costa CTC, Souza JAL. Ovicidal and larvicidal activity in vitro of Spigelia anthelmia Linn. extracts on Haemonchus contortus. Vet Parasitol. 2003 Nov;117(1-3):43-9,doi:10.1016/j.vetpar.2003.07.021

38. Ntalli NG, Ferrari F, Giannakou I, Menkissoglu-Spiroudi U. Phytochemistry and nematicidal activity of the essential oils from 8 greek Lamiaceae aromatic plants and 13 terpene components. J Agric Food Chem. 2010 Jun;58(13):7856-63,doi:10.1021/jf100797m

39. Kloster FS. Avaliação in vitro de óleos essenciais como anti-helmíntico de bovinos [dissertação]. Curitiba: Universidade Federal do Paraná; 2013. 145 p.

40. Camurça-Vasconcelos LF, Melo LM, Nascimento NRF, Teixeira PGM, Menezes DB, Silva RA, Souza IP, Queioz MGR, Morais SM, Bevilaqua CML. Avaliação toxicológica do extrato acetato de etila de Spigelia anthelmia Linn, em ratos e camundongos. Rev Bras Ci Vet. 2004 Set/Dez;11(3):159-62,doi: 10.4322/rbcv.2014.369 